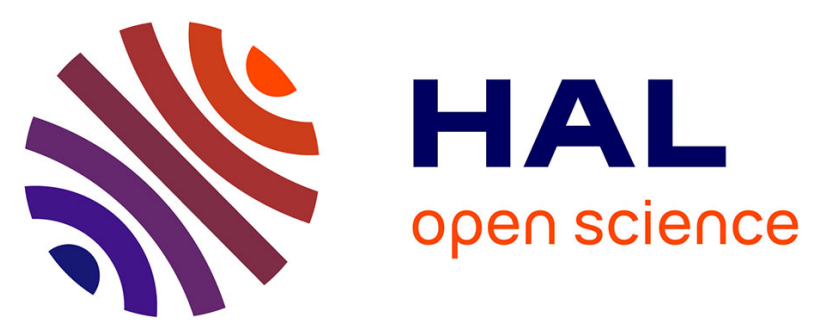

\title{
Space-Time Plasma Steering Source: Synthesis and Outlooks
}

\author{
V Mazières, R Pascaud, L Stafford, P Bonnet, A Al Ibrahim, C Chauvière, \\ Laurent Liard, Simon Dap, Richard Clergereaux, Olivier Pascal
}

\section{- To cite this version:}

V Mazières, R Pascaud, L Stafford, P Bonnet, A Al Ibrahim, et al.. Space-Time Plasma Steering Source: Synthesis and Outlooks. XXXIV General Assembly and Scientific Symposium (GASS) of the International Union of Radio Science (Union Radio Scientifique Internationale-URSI), Aug 2021, Rome, Italy. pp.1-4, 10.23919/URSIGASS51995.2021.9560460 . hal-03373220v2

HAL Id: hal-03373220

https://hal.science/hal-03373220v2

Submitted on 18 Oct 2021

HAL is a multi-disciplinary open access archive for the deposit and dissemination of scientific research documents, whether they are published or not. The documents may come from teaching and research institutions in France or abroad, or from public or private research centers.
L'archive ouverte pluridisciplinaire HAL, est destinée au dépôt et à la diffusion de documents scientifiques de niveau recherche, publiés ou non, émanant des établissements d'enseignement et de recherche français ou étrangers, des laboratoires publics ou privés. 


\title{
Space-Time Plasma Steering Source: Synthesis and Outlooks
}

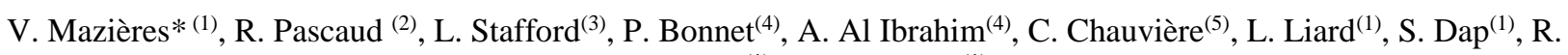 \\ Clergereaux $^{(1)}$ and O. Pascal ${ }^{(1)}$
}

(1) Laboratoire LAPLACE, Université Paul Sabatier, 118 route de Narbonne, 31062 Toulouse, France

(2) ISAE-SUPAERO, Université de Toulouse, 10 avenue Edouard Belin, 31055 Toulouse France

(3) Département de physique, Université de Montréal, Montréal, Québec H3C 3J7, Canada

(4) CNRS, SIGMA Clermont, Institut Pascal, Université Clermont Auvergne, Clermont-Ferrand, France

(5) CNRS, UMR 6620, Laboratoire de Mathématiques, Clermont-Ferrand, France

\begin{abstract}
The aim of this paper is to present the promising outlooks that lies ahead for the innovative plasma source we introduced recently. The idea of this source is to dynamically control the plasma location by changing the waveform of the transmitted signal to an overmoded cavity. It is then the behavior of the waves inside the cavity that dynamically control the plasma location, hence the name of Space-Time Plasma Steering Source. Since its introduction in 2019 , we have produced three main papers, aimed at different scientific communities: applied physics, physics of plasma, signal processing and mathematical and numerical methods. An overview of these works is first presented in this paper. Then, the promising outlooks for this unusual plasma source are identified and classified into four domains, among which the most important and essential one being the unhooking of the plasmas from the initiators in order to control their location anywhere in the cavity. Several solutions for this "unhooking issue" are then proposed, the most straightforward one being the use of non-intrusive probes.
\end{abstract}

\section{Introduction}

Plasma, or ionized gas, is a very interesting medium with various and exotic physical properties. It finds applications in different area of daily life and industry. The key issue to ignite a plasma is to obtain an electric field high enough to trigger an electron avalanche phenomenon in a medium. There exist a lot of methods to do it, which are based on quite different technologies [1].

One of the challenges that face these plasma technologies is the development of "plasma source concepts that can be scaled to larger dimensions" [1]. Indeed, there is an increasing need to be able to process large area which comes, among other things, from the need to process solar panel, flat panel displays, and larger wafers [1].

However, in material processing, all of these technologies share a common feature: the more the area to be processed is large, the more it is difficult to process it. In fact, when increasing the area these technologies face physical and technological limitations $[1,2,3,4]$.

Microwave plasmas are usually ignited by exciting a resonance mode of a metallic chamber whose geometry is specifically designed to obtain a plasma discharge at the desired location [5]. However, when the cavity dimensions are increased, it becomes overmoded and the control of the spatial distribution of the electric field (and thus of the plasma) is very difficult with these technologies [2].

To address this challenge of controlling plasmas in large cavities (overmoded), we proposed in 2019 an innovative concept of microwave plasma source [6], that we name in this paper "Space-Time Plasma Steering Source" (STPSS). Contrary to the existing microwave plasma technologies, the idea is not to generate a plasma at a fixed location, but to ignite a plasma whose location can be dynamically controlled in a large cavity. To do that, one need to unlock a usually fixed parameter, namely the temporal shape of the exciting signal, which will be more complex than a continuous wave. It then becomes an additional degree of freedom of the system.

This paper is organized as follow. First we summarize the experimental results that we obtained since our pioneer paper of 2019 [6]. We will show how the STPSS was first developed using Time Reversal (TR) [6,7] and then using a more elaborated technique: the Linear Combination of Configuration Field (LCCF) [8]. Then we will discuss about promising outlooks for this innovative plasma source, the main and essential one being the "unhooking" of the plasma from the initiators.

\section{Concept of the Space-Time Plasma Steering Source}

The concept of the Space-Time Plasma Steering Source is presented on Figure 1. It consists in dynamically controlling the position of the plasma in a cavity by playing on the waveform of the transmitted signal to the cavity. Indeed, this waveform determines the behavior of the waves inside the cavity and so the spatio-temporal structure of the field. In order to ignite a plasma at a desired location, the waveform must be controlled in order to obtain an intensification of the electric field at this location. This intensification will be obtained by making the waves constructively interfere at this location. Then, the waveform must be changed so that the waves will constructively interfere at the next desired location, to obtain the plasma at this novel location.

To reach this objective we can see that it is necessary to use an elaborate technique for controlling the waves, allowing the spatio-temporal control of the field inside the cavity. Furthermore, this technique must involve a 
phenomenon of intensification of the field at the desired time and position to ignite a plasma. We will see in the next section that Time Reversal (TR) fully meets these criteria and allows to ignite and control plasmas in cavity.

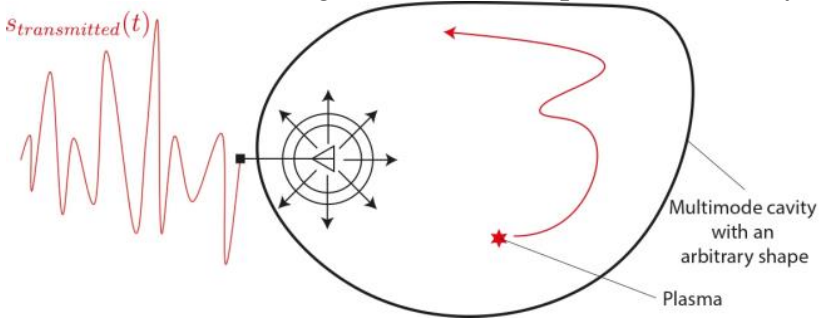

Figure 1. Principle of the STPSS. The location of the plasma is controlled by the waveform of the transmitted signal $s_{\text {transmitted }}(t)$ to an overmoded cavity, which dynamically shapes the field in the cavity.

\section{Experimental demonstrations of the Space-Time Plasma Steering Source}

The experimental implementation of the STPSS requires in practice two phases. One first phase, the "probing phase", during which the behavior of the waves is evaluated between the exciting antenna and the desired location (at which we want to ignite a plasma). Then, during the second phase, also known as the "emitting phase", the transmitted signal (constructed based on the information collected during the first step) must allow the ignition of the plasma at the desired location.

Our experimental setup consists of a main cavity of $0.6 \times$ $0.6 \times 0.3 \mathrm{~m}^{3}$ - that is to say $5 \lambda_{c} \times 5 \lambda_{c} \times 2.5 \lambda_{c}$ at our working microwave carrier frequency of about $f_{c}=$ $2.4 \mathrm{GHz}$ - in which the pressure and the gas can be controlled (typically with argon at 1 torr) and an appendix in which the air remains at atmospheric pressure to prevent gas breakdown in its vicinity during the high power emission of the complex waveforms (see Figure 2). This cavity includes several antennas: one of dimension $\lambda_{c} / 4$ located in the appendix and the other ones located in the main cavity. The antennas of the main cavity (which are electrically small metallic monopoles), act as emitting antennas during the probing phase of the STPSS process. During the emitting phase, they act as receiving antennas (allowing a measure of the field evolution at their locations) as well as initiators for plasma breakdown, as they produce a local enhancement of the electric field. Hence, they will be referred to as "initiator" in the following of this paper.

The aim of our experiments is to control the plasma location on the initiators by playing on the waveform of the signal transmitted to the antenna in the appendix.

\subsection{Space-Time Plasma Steering Source based on Time reversal}

The first wave control technique that we used as a SpaceTime Plasma Steering Source is TR [6]. It has been shown to allow to focus electromagnetic waves [9]. In a classical TR experiment, the probing phase consists in sending a short pulse that propagates through a complex medium, and the radiated field is recorded using an array of transducers, whose number can be reduced down to only one in a large reverberant chaotic cavity [10]. The recorded waveforms are then time-reversed and reemitted by this transducer such that the resulting wave converges back to its initial point of emission. An interesting property of TR for our application is that its focusing capability increases with the cavity volume, so large overmoded cavities are useable.

The aim of our experiments is to focus the electromagnetic waves by TR on initiators 1,2 , or 3 represented on Figure 2 (with antenna 4 used as a transmitting antenna) in order to obtain an electric field high enough to reach breakdown conditions.

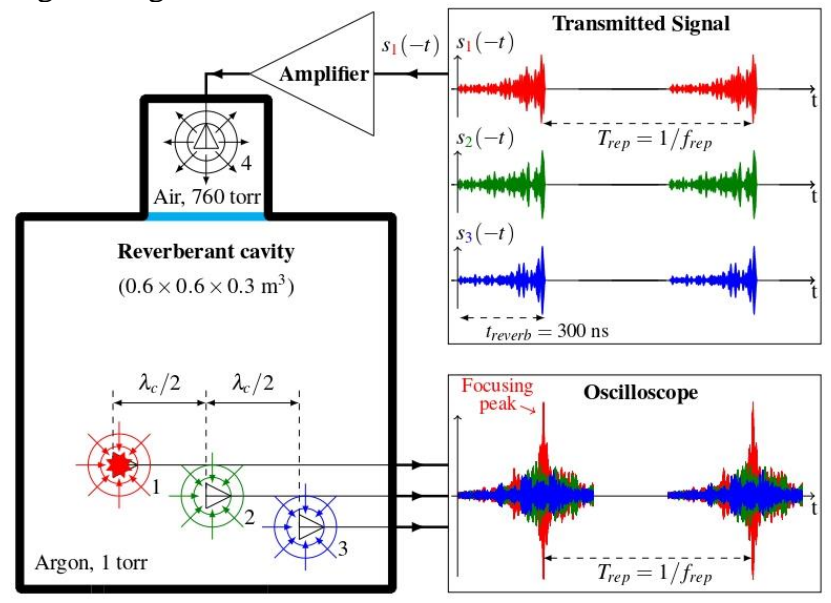

Figure 2. Sketch of an experiment of plasma ignition by TR on initiator 1 (the presence of the plasma is denoted by the red star) [6].

In our experiments, the probing phase of TR is carried out in the frequency domain (with a Vector Network Analyzer) between antenna 4 and each of the three initiators 1, 2, and 3. Then using signal post-processing techniques, the impulse response $s_{1}(t), s_{2}(t)$, and $s_{3}(t)$ between antenna 4 and initiators 1,2 , and 3 respectively are constructed. They correspond to impulse response with an impulse of $8 \mathrm{~ns}$ (modulated at a carrier frequency $f_{c}=2.4 \mathrm{GHz}$ ), which is the shortest pulse that we are able to generate with our experimental setup [6] and possess a reverberation time of about $t_{\text {reverb }}=300 \mathrm{~ns}$. Then, emission of the time reversed signals $s_{1}(-t), s_{2}(-t)$, or $s_{3}(-t)$ at antenna 4 yields a focusing of the electromagnetic waves on initiator 1, 2, or 3 respectively. In the results presented in this subsection, the signal $s_{1}(-t), s_{2}(-t)$ or $s_{3}(-t)$ is amplified (using a $2 \mathrm{~kW}$ amplifier) and transmitted periodically to the cavity with a repetition period of $T_{r e p}=166 \mu \mathrm{s}$. The pressure inside the main cavity is of 1 torr in argon, which are conditions that allows to ignite plasmas with our experimental setup [6]. An example of our experimental setup used to ignite a plasma on initiator 1 is showed on Figure 2. The signals measured with an oscilloscope on initiators 1, 2 and 3 are plotted in red, green and blue respectively. We see that we obtain a focusing peak of $8 \mathrm{~ns}$ on initiator 1 and no peak on both initiators 2 and 3 , as desired. 
Pictures of the initiators 1, 2, and 3 are shown on Figure 3 (top picture). The three bottom pictures show the plasmas ignited when sending $s_{1}(-t), s_{2}(-t)$, and $s_{3}(-t)$. This demonstrates that TR can be used as a STPSS, as it allows to control the plasma location in the overmoded cavity by changing the waveform of the transmitted signal [6]. Note that for now these plasmas have only been ignited and controlled on initiators, which are necessary for the probing phase.

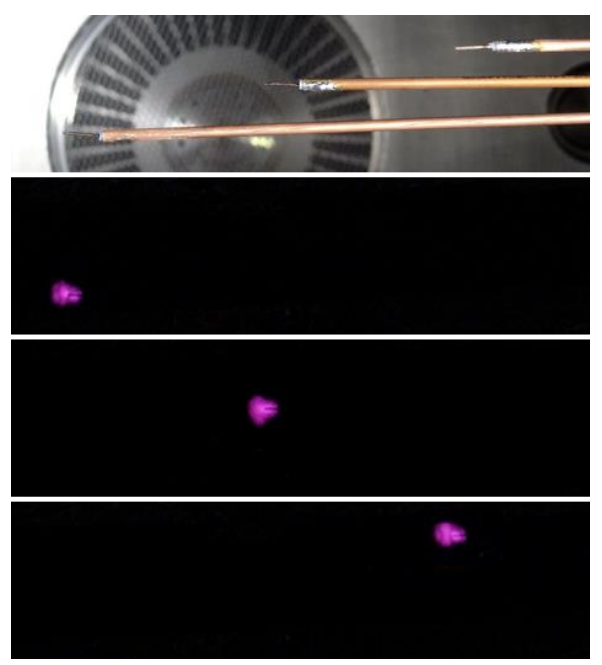

Figure 3. Picture of the three initiators 1, 2, and 3 of Figure 2 (top) and of the ignited plasmas by TR [6].

\subsection{Spatio-temporal description of the STPSS based on Time Reversal}

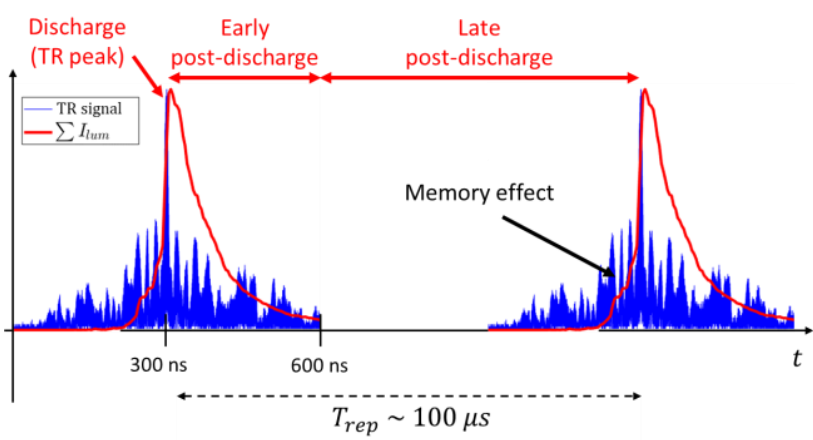

Figure 4. Representation of the three time intervals: discharge, early post-discharge and late post-discharge. In blue is represented the absolute value of the TR signal and in red the integrated light intensity as a function of time.

The TR plasma source has quite unusual characteristics:

- Plasma controlled in a overmoded cavity.

- Plasma dimensions and position are uncorrelated from the cavity design and dimensions.

- Very low rise time (of about $1 \mathrm{~ns}$ ).

- Very low duty cycle $\sim 0.05$, maintaining the discharges. In order to investigate this unusual source, in addition to the electrical measurements presented in the previous subsection, optical diagnostics of the plasma ignited by TR on one of the three monopoles have also been performed. It consists in imaging using a camera that enables to measure the spatio-temporal evolution of the light intensity emitted by the plasma during the signal period. These investigations have been made as a function of the pressure $p$ and the repetition period of the TR pulses $T_{\text {rep }}$ in our paper [7] and three temporal intervals, with distinct mechanisms, have been identified: the discharge, the "early post-discharge" and the "late postdischarge", as represented on Figure 4. The interested reader must refer to [7] to fully grasp these works.

\subsection{Space-Time Plasma Steering Source based on LCCF}

The second wave control technique that we used as a STPSS is the LCCF method [8]. This technique allows to determine a temporal waveform that leads to predefined electromagnetic fields at one or more positions over a given interval of time (contrary to TR that allows only the control of the field at the time and location of the focusing). The probing phase is the same as the one of a TR experiment, meaning the impulse responses are recorded between the emitting point and the receiver points. Then, the LCCF finds a linear combination of these responses to compute the desired waveform. Emission of this waveform yields the desired target electromagnetic fields, and this for an arbitrary duration [11]. We have used this technique to improve the control of the plasmas in cavity [8].

\section{Outlooks}

\subsection{Unhooking issue}

The most important and essential step that needs to be taken is the "unhooking" of the plasma from the initiators, in order to obtain a "ball" of plasma that could be moved anywhere in the overmoded cavity. The difficulty lies in evaluating, during the probing phase, the behavior of the waves between the exciting antenna and the location at which we want to ignite a plasma. Indeed, removing an antenna (initiator) from the cavity changes significantly the behavior of the waves. Thus the information collected during the probing phase does not hold during the emitting phase if an antenna (initiator) is removed, so no TR focusing would occur. To address this issue, several ways can be envisioned:

- The probing phase could be obtained by simulating the behavior of the waves in the cavity. This would be very complicated as it would require an accurate representation of the experimental cavity and long simulation times [12].

- We could also think about using elaborated techniques for controlling the waves, in order for example to interpolate the information of the behavior of the waves between two initiators.

- The more promising and straightforward way would be the use of a non-intrusive probe. A lot of work have been done on TR with electro-optic probes at microwave frequencies in reverberant room [13]. Thus, the use of an electro-optic probe to "unhook" the plasma from the initiators seems to be very promising. 


\subsection{Plasma diagnostics}

In order to understand the rich physics of these unusual plasmas, further characterizations are planned in the future, for example using a time resolved spectrometer.

\subsection{Modeling of the multi-physics problem}

It would be useful to be able to describe theses plasmas ignited by TR with a numerical model, as it would help in grasping the physical mechanisms and it would allow to follow quantities that are not easily accessible experimentally, such as the electron density. The difficulties lie in the multi-physics modelling, along with the long simulation times that are required.

Another interesting aspect would be a more fundamental study of the physics of these unusual plasmas. A tool seems to lend itself to this purpose: the space-time diagrams. A good example of how these diagrams can be effectively used to give "visual perspective of the overall physics" is found in [14] for describing space-time metamaterials. As a plasma ignited with a Space-Time Plasma Steering Source consists a priori both of a timediscontinuity (the plasma is ignited at the focusing time) and a space-discontinuity (the plasma is spatially localized), space-time diagram seems to be the right tool for describing the physics of these plasmas.

\subsection{Potential applications}

We already mentioned that this innovative source could really be useful in surface processing as it would allow large area processing in overmoded cavities.

Another potential application could be found at higher pressure, for example for microwave plasma assisted combustion and ignition as proposed in [15].

The ability to control in space and time the plasmas suggests also interesting outlooks in the field of spacetime modulated media [14].

\section{Conclusion}

This paper summarizes the work we have done these last few years on the new plasma source concept, the SpaceTime Plasma Steering Source, and propose the interesting and challenging outlooks for this exotic plasma source. Further work on each of these outlooks, especially on the "unhooking issue", is planned in the future.

\section{Acknowledgements}

The authors would like to acknowledge the DGA/AID for their funding support and the CEA-Gramat for the material support. The first author would also like to acknowledge the CNES for their postdoctoral funding.

\section{References}

1. Seiji Samukawa et al, "The 2012 Roadmap", J. Phys. D: Appl. Phys. 45.25, juin 2012, p. 2530012, doi: $10.1088 / 0022-3727 / 45 / 25 / 253001$.
2. G. S.J. Sturm et al, "Microwaves and microreactors: Design challenges and remedies", Chemical Engineering Journal, Volume 243, 2014, p. 147-158, ISSN 1385-8947, doi :10.1016/j.cej.2013.12.088.

3. Yong-Xin Liu and Yu-Ru Zhang, "Electromagnetic effects in high-frequency large-area capacitive discharges", Journal of Vacuum Science \& Technology, A 33, 020801 (2015), doi: 10.1116/1.4907926.

4. M.A. Lieberman et al, "Standing wave and skin effects in large-area, high-frequency capacitive discharges", Plasma Sources Sci. Technol., 11283 (2002), doi: 10.1088/0963-0252/11/3/310.

5. J. Achard et al, "High quality MPACVD diamond single crystal growth: High microwave power density regime," J. Phys. D, 40, 6175-6188 (2007), doi: 10.1088/0022-3727/40/20/S04.

6. V. Mazières, et al, "Plasma generation using time reversal of microwaves", Applied Physics Letters, 115.15 (oct. 2019), p. 154101, doi: 10.1063/1.5126198.

7. V. Mazières et al, "Spatio-temporal dynamics of a nanosecond pulsed microwave plasma ignited by time reversal", Plasma Sources Sci. Technol., 29 125017, 2020, doi: 10.1088/1361-6595/abc9ff.

8. V. Mazières et al., "Transient Electric Field Shaping With the Linear Combination of Configuration Field Method for Enhanced Spatial Control of Microwave Plasmas", IEEE Access, 8 (2020), p. 177084-177091, doi: 10.1109/ACCESS.2020.3025366.

9. G. Lerosey et al, "Time reversal of electromagnetic waves," Phys. Rev. Lett. 92, 193904 (2004), doi: 10.1103/PhysRevLett.92.193904

10. C. Draeger and M. Fink., "One-Channel Time Reversal of Elastic Waves in a Chaotic 2D-Silicon Cavity", Phys. Rev. Lett. 79.3 (juil. 1997), p. 407-410, doi: 10.1103/PhysRevLett.79.407

11. J. Benoit et al, "Time-dependent current source identification for numerical simulations of Maxwell's equations,", J. Com-put. Phys., vol. 289, pp. 116-128, May 2015, doi: 10.1016/j.jcp.2015.02.033.

12. Bo Xiao et al., "Focusing waves at arbitrary locations in a ray-chaotic enclosure using time-reversed synthetic sonas", Phys. Rev. E, 93.5 (mai 2016), doi: 10.1103/PhysRevE.93.052205.

13. F. Monsef and A. Cozza, "Analysis of time-reversalbased propagation for spatial focusing and multiplexing," IEEE-APS Topical Conference on Antennas and Propagation in Wireless Communications, Torino, 2011, pp. 827-830, doi: 10.1109/APWC.2011.6046804.

14. C. Caloz; Z.-L. Deck-Léger, "Spacetime metamaterials", arXiv:1905.00560.

15. B. Fragge et al, "Etude de l'allumage d'un carburant liquide par des micro-onde focalisées", JCMM 2020 $16^{\text {èmes }}$ Journées de Caractérisation Microondes et Matériaux, Toulouse, 2020. 